\title{
Gasdermin D Exerts Anti-inflammatory Effects by Promoting Neutrophil Death
}

\section{Citation}

Kambara, H., F. Liu, X. Zhang, P. Liu, B. Bajrami, Y. Teng, L. Zhao, et al. 2018. “Gasdermin D Exerts Anti-inflammatory Effects by Promoting Neutrophil Death." Cell reports 22 (11): 2924-2936. doi:10.1016/j.celrep.2018.02.067. http://dx.doi.org/10.1016/j.celrep.2018.02.067.

\section{Published Version}

doi:10.1016/j.celrep.2018.02.067

\section{Permanent link}

http://nrs.harvard.edu/urn-3:HUL.InstRepos:35982116

\section{Terms of Use}

This article was downloaded from Harvard University's DASH repository, and is made available under the terms and conditions applicable to Other Posted Material, as set forth at http:// nrs.harvard.edu/urn-3:HUL.InstRepos:dash.current.terms-of-use\#LAA

\section{Share Your Story}

The Harvard community has made this article openly available.

Please share how this access benefits you. Submit a story.

Accessibility 
Published in final edited form as:

Cell Rep. 2018 March 13; 22(11): 2924-2936. doi:10.1016/j.celrep.2018.02.067.

\title{
Gasdermin D Exerts Anti-inflammatory Effects by Promoting Neutrophil Death
}

\author{
Hiroto Kambara ${ }^{1,2,7}$, Fei Liu ${ }^{3,7}$, Xiaoyu Zhang ${ }^{1,2,3,7}$, Peng Liu ${ }^{3}$, Besnik Bajrami ${ }^{4}$, Yan \\ Teng $^{1,2}$, Li Zhao ${ }^{1,2}$, Shiyi Zhou ${ }^{1,2}$, Hongbo $\mathrm{Yu}^{5}$, Weidong Zhou ${ }^{6}$, Leslie E. Silberstein ${ }^{1,2}$, Tao \\ Cheng $^{3}$, Mingzhe $\mathrm{Han}^{3}$, Yuanfu $\mathrm{Xu}^{3}$, and Hongbo R. Luo ${ }^{1,2,8,{ }^{*}}$ \\ ${ }^{1}$ Department of Pathology, Harvard Medical School, Dana-Farber/Harvard Cancer Center, \\ Boston, MA 02215, USA \\ 2Department of Laboratory Medicine, Children's Hospital Boston, Enders Research Building, \\ Room 814, Boston, MA 02115, USA \\ ${ }^{3}$ The State Key Laboratory of Experimental Hematology, Institute of Hematology and Blood \\ Diseases Hospital, Chinese Academy of Medical Sciences and Peking Union Medical College, \\ 288 Nanjing Road, Tianjin 300020, China \\ ${ }^{4}$ Center for Development of Therapeutics, Broad Institute, 415 Main Street, Cambridge, MA \\ 02142, USA \\ 5VA Boston Healthcare System, Department of Pathology and Laboratory Medicine, Harvard \\ Medical School, 1400 VFW Parkway, West Roxbury, MA 02132, USA \\ ${ }^{6}$ Center for Applied Proteomics and Molecular Medicine, George Mason University, Manassas, VA \\ 20110, USA
}

\section{SUMMARY}

Gasdermin D (GSDMD) is considered a proinflammatory factor that mediates pyroptosis in macrophages to protect hosts from intracellular bacteria. Here, we reveal that GSDMD deficiency paradoxically augmented host responses to extracellular Escherichia coli, mainly by delaying neutrophil death, which established GSDMD as a negative regulator of innate immunity. In contrast to its activation in macrophages, in which activated inflammatory caspases cleave GSDMD to produce an N-terminal fragment (GSDMD-cNT) to trigger pyroptosis, GSDMD

This is an open access article under the CC BY-NC-ND license (http://creativecommons.org/licenses/by-nc-nd/4.0/).

${ }^{*}$ Correspondence: hongbo.luo@ childrens.harvard.edu.

${ }_{8}^{7}$ These authors contributed equally

${ }^{8}$ Lead Contact

\section{SUPPLEMENTAL INFORMATION}

Supplemental Information includes Supplemental Experimental Procedures, seven figures, and four movies and can be found with this article online at https://doi.org/10.1016/j.celrep.2018.02.067.

AUTHOR CONTRIBUTIONS

Conceptualization, H.R.L. and H.K.; Methodology, F.L., X.Z., P.L., and H.K.; Investigation, F.L., X.Z., B.B., Y.T., L.Z., S.Z., H.Y., W.Z. and H.K.; Writing: Original Draft, H.K.; Writing: Review and Editing, H.K., F.L., X.Z., Y.X., L.E.S., and H.R.L.; Funding Acquisition, T.C., F.L., Y.X., L.E.S., and H.R.L.; Resources, T.C., M.H., Y.X., and L.S.; Supervision, T.C., M.H., Y.X., L.S., and H.R.L.

DECLARATION OF INTERESTS

The authors declare no competing interests. 
cleavage and activation in neutrophils was caspase independent. It was mediated by a neutrophilspecific serine protease, neutrophil elastase (ELANE), released from cytoplasmic granules into the cytosol in aging neutrophils. ELANE-mediated GSDMD cleavage was upstream of the caspase cleavage site and produced a fully active ELANE-derived NT fragment (GSDMD-eNT) that induced lytic cell death as efficiently as GSDMD-cNT. Thus, GSDMD is pleiotropic, exerting both pro- and anti-inflammatory effects that make it a potential target for antibacterial and antiinflammatory therapies.

\section{In Brief}

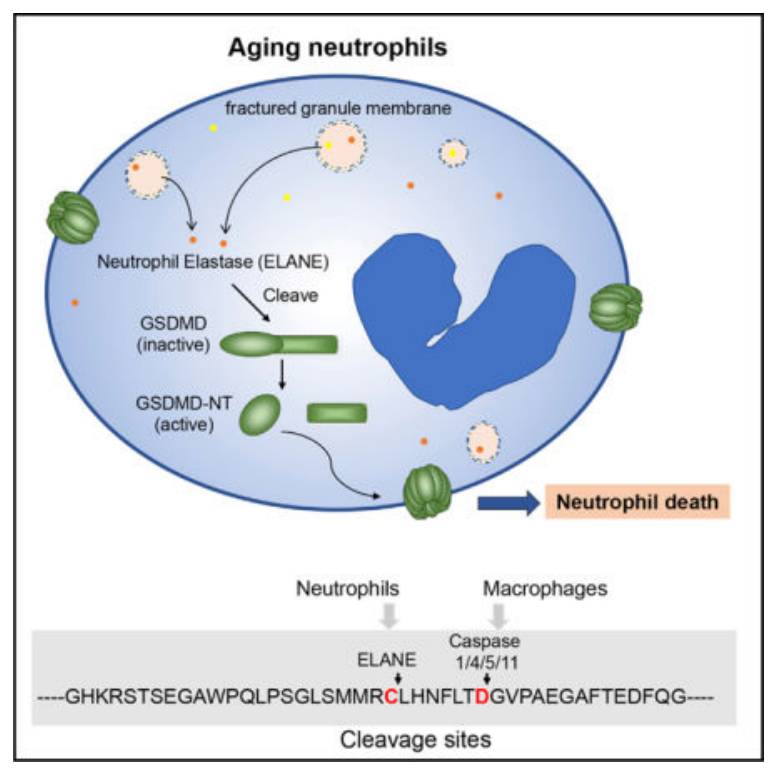

Kambara et al. find that GSDMD deficiency augments host responses to extracellular Escherichia coli, mainly by delaying neutrophil death, establishing GSDMD as a negative regulator of innate immunity. GSDMD cleavage and activation in neutrophils is mediated by ELANE, released from cytoplasmic granules into the cytosol in aging neutrophils.

\section{INTRODUCTION}

Neutrophils are terminally differentiated cells and have a short lifespan in circulation and tissue (Lahoz-Beneytez et al., 2016; Lord et al., 2001). Neutrophil death plays a critical role in regulating neutrophil numbers in infection and inflammation. Aging neutrophils undergo programmed death and are then recognized, engulfed, and safely cleared by professional phagocytes such as macrophages (Savill et al., 1989). Neutrophil death shares many features of classical apoptosis and involves caspase activation. However, it is not entirely caspase dependent. Inhibition of caspases, which are critical mediators of apoptosis, delays but does not completely abolish cell death, suggesting that other mechanisms of neutrophil death exist Loison et al., 2014; Luo and Loison, 2008).

Gasdermin D (GSDMD) was recently identified as the factor responsible for the inflammatory form of lytic pyroptotic cell death, a critical antibacterial innate immune 
defense mechanism (He et al., 2015; Kayagaki et al., 2015; Shi et al., 2015a). Activated inflammatory caspases (caspase-1/4/5/11) cleave GSDMD in macrophages to produce an Nterminal fragment (GSDMD-cNT) that triggers pyroptosis by binding to phosphatidylinositol phosphates and phosphatidylserine in the cell membrane inner leaflet to induce membrane pore formation and interleukin-1 $\beta$ (IL-1 $\beta$ ) secretion (Aglietti et al., 2016; Chen et al., 2016; Ding al., 2016; He et al., 2015; Liu et al., 2016; Sborgi et al., 2016). GSDMD-cNT also binds to cardiolipin in the inner and outer leaflets of bacterial membranes, suggesting that it may directly kill bacteria by perforating bacterial membranes (Ding al., 2016; Liu et al., 2016). Based on these studies, GSDMD generally considered proinflammatory and an important host response to bacterial infection. Although GSDMDdeficient mice are protected from lethal endotoxemia (Kayagaki et al., 2015; Shi et al., 2015a), their bactericidal capability against extracellular pathogens has not been examined directly.

Here, we reveal that GSDMD deficiency unexpectedly and paradoxically augments host defenses against extracellular Escherichia coli by delaying neutrophil death. Its cleavage and activation in neutrophils is caspase independent, instead mediated by a neutrophil-specific serine protease, neutrophil elastase (ELANE), and released from neutrophil granules into the cytosol during neutrophil death. GSDMD controls neutrophil death and negatively regulates neutrophil-mediated innate immunity. Thus, GSDMD can exert context-dependent proinflammatory and anti-inflammatory effects and is a unique target for antibacterial and anti-inflammatory therapies.

\section{RESULTS}

\section{GSDMD-Deficient Mice Display Augmented Bactericidal Activity against E. coli}

Pyroptosis often occurs when macrophages are infected with intracellular pathogens and is thought to be critical for the clearance of these pathogens (Jorgensen and Miao, 2015; Jorgensen et al., 2016). Whether GSDMD contributes to the general bactericidal activity in host defense and the potential mechanism leading to its activation during this process remains elusive. We therefore explored bacterial killing in wild-type (WT) and GSDMDdeficient mice (Figures S1A-S1E) in a murine acute peritoneal infection (peritonitis) model (Li et al., 2009). As previously reported, $G s d m d^{-1-}$ mice were viable, fertile, and of normal size; peripheral blood differential counts were normal; and blood smears showed no hematopoietic abnormalities (Figures S1F-S1H). We then assessed the survival of WT and knockout (KO) mice in a lipopolysaccharide (LPS)-induced sepsis model. Consistent with the published results, our GSDMD-deficient mice also showed significantly improved survival compared to the WT mice (Figure S1I).

In this model, the host defenses suppress bacterial proliferation and gradually clear the intraperitoneally injected Gram-negative E. coli. $36 \mathrm{hr}$ after injection of $5 \times 10^{7}$ live E. coli, the number of viable bacteria in peritoneal cavities consistently reduced to $2 \times 10^{4}$ in WT mice, reflecting a functioning immune system. Significantly fewer bacteria $\left(1.8 \times 10^{3}\right)$ were detected in inflamed GSDMD-deficient mice, suggesting that host responses to bacteria are enhanced in these mice (Figure 1A). Bacteria disseminate widely in infected hosts during severe infection, but, consistent with enhanced host immune responses in GSDMD-deficient 
mice, bacterial loads also were significantly reduced in the spleens, livers, and kidneys of GSDMD-deficient mice compared to WT mice (Figure 1B). WT mice also displayed marked splenomegaly, another sign typical of bacterial infection (Figures S1J and S1K). Infection-associated splenomegaly was alleviated significantly in the GSDMD-deficient mice. Collectively, GSDMD disruption appears to augment host bactericidal activity to $E$. coli infection, indicating that GSDMD may be a negative regulator of innate immunity.

\section{The Augmented Host Antibacterial Response in GSDMD-Deficient Mice Is the Result of Enhanced Neutrophil Accumulation}

Enhanced bacterial killing may be the result of enhanced phagocytosis of bacteria by neutrophils. We first assessed neutrophil phagocytosis capability using mouse bone marrow neutrophils. GSDMD deficiency did not alter phagocytosis efficiency as measured by the percentage of neutrophils that engulfed at least one E. coli bioparticle (Figure 2A). The phagocytosis index of GSDMD-deficient neutrophils also was comparable to that of WT neutrophils: nearly 100 bacteria were engulfed by 100 neutrophils (Figure 2A). We next examined whether GSDMD disruption intrinsically alters the bactericidal capability of neutrophils in vitro using purified WT and GSDMD-deficient neutrophils, but GSDMDdeficient neutrophils were equally as efficient as WT neutrophils at clearing live $E$. coli (Figure 2B). The intracellular bactericidal activity of neutrophils was further assessed by treating samples with a plasma membrane-impermeable antibiotic that kills extracellular bacteria but not internalized intracellular bacteria. The same number of viable engulfed bacteria was detected in both GSDMD-deficient and WT neutrophils, suggesting that GSDMD does not directly regulate the killing of intracellular bacteria (Figure 2B). Thus, the increased bactericidal activity observed in GSDMD-deficient mice does not appear to occur because the intrinsic bacterial killing capability of each neutrophil is enhanced. Similar results were observed when phagocytosis and bacterial killing capability were examined in peripheral blood neutrophils (Figures 2C and 2D).

Elevated bacterial killing also may be caused by enhanced neutrophil accumulation in the inflamed peritoneal cavity. Few peritoneal neutrophils were present in unchallenged mice; however, after $E$. coli injection, the number of peritoneal neutrophils increased to the same extent in both WT and GSDMD-deficient mice to nearly $9 \times 10^{6}$ after $24 \mathrm{hr}$ (Figure 2E). GSDMD deficiency does not, therefore, affect initial neutrophil recruitment, but GSDMDdeficient mice did show drastic increases in E. coli-induced neutrophil accumulation (15 $\times$ $10^{6}$ versus $7 \times 10^{6}$ neutrophils in $\mathrm{Gsdm}^{-1-}$ versus WT mice, respectively) $48 \mathrm{hr}$ after E. coli injection (Figure 2E). Therefore, the augmented host antibacterial response in GSDMDdeficient mice is likely to be the result of enhanced neutrophil accumulation in the inflamed peritoneal cavity rather than an enhanced intrinsic capacity of neutrophils to kill bacteria. Thus, under this condition, GSDMD acted as an anti-inflammatory factor. Consistently, the levels of proinflammatory cytokines in the peritoneal cavity were elevated significantly in Gs $\mathrm{md}^{-1-}$ mice, suggesting that the production of these cytokines was not mediated mainly by macrophage pyroptosis (Figure $2 \mathrm{~F}$ ). 


\section{Delayed Neutrophil Death Led to Elevated Neutrophil Accumulation and Enhanced Host Defenses in Gsdmd/Mice}

Elevated neutrophil accumulation at sites of infection can occur simply as a result of enhanced neutrophil recruitment from the circulation to the inflamed peritoneal cavity, but the unaltered peritoneal neutrophil counts during the early stage of bacterial infection make this unlikely. In addition, we measured the peripheral neutrophil count before and after infection (Figure S2A). No difference was detected between the WT and GSDMD-deficient mice. In the bacterial peritonitis model, neutrophil recruitment can be regulated indirectly by the number of live bacteria remaining in the peritoneal cavity, which was reduced in GSDMD-deficient mice. Thus, to directly assess neutrophil recruitment during inflammation, we induced sterile peritonitis with thioglycolate (TG), which more rapidly elicited neutrophil recruitment than the bacterial model to reach a peak $7 \mathrm{hr}$ after injection (Li et al., 2011). Again, similar numbers of peritoneal neutrophils were detected in WT and GSDMD-deficient mice at this early phase of inflammation, confirming that GSDMD deficiency does not alter neutrophil recruitment (Figures 3A and 3B). Furthermore, we measured the number of both resident and inflammatory macrophages in the peritoneal cavity. No difference was detected between the WT and GSDMD-deficient mice (Figure S2B). In addition, using an in vitro assay we examined the clearance of dead neutrophils by macrophages, a process known as efferocytosis (Poon et al., 2014). GSDMD disruption in macrophages did not affect efferocytosis efficiency (Figure S2C). We therefore speculated that the elevated neutrophil accumulation during late-stage infection was caused by a prolonged lifespan of recruited neutrophils.

We next explored whether neutrophil death is delayed in the peritoneal cavities of GSDMDdeficient mice. We measured the percentage of dying neutrophils in peritoneal lavage fluid (PLF) by fluorescence-activated cell sorting (FACS) analysis (Figure 3C, S2D, and S3E). Although apoptotic neutrophils can be engulfed and cleared by macrophages, there were still $>20 \%$ dying neutrophils (propidium iodide-positive $\left[\mathrm{PI}^{+}\right]$cells) in the inflamed peritoneal cavities of WT mice $48 \mathrm{hr}$ after $E$. coli injection compared to only $8 \%$ dying neutrophils in GSDMD-deficient mice. Notably, the decrease of dying neutrophil number in GSDMDdeficient mice was already detectable at $24 \mathrm{hr}$ after $E$. coli injection before the total neutrophil number had started to decline (Figure 3D). Delayed neutrophil death may, therefore, be responsible for augmented neutrophil accumulation in GSDMD-deficient mice.

A reduction in dying peritoneal neutrophils could be the result of an altered peritoneal inflammatory environment, exaggerated clearance by peritoneal macrophages, or both. We next used a well-established in vitro system to determine whether delayed in vivo neutrophil death was indeed caused by alterations in intrinsic death/survival pathways in GSDMDdeficient neutrophils (Xu et al., 2010) (Figure S3A). Neutrophils die even in the absence of any stimuli. Thus, this type of death also is called neutrophil spontaneous or constitutive death. We first explored whether neutrophil constitutive death is delayed in a GSDMDdeficient neutrophil population. Freshly isolated GSDMD-deficient bone marrow or peripheral blood neutrophils were morphologically normal (Figure S3B). However, consistent with the in vivo results, in vitro-cultured GSDMD-deficient bone marrow neutrophils showed delayed spontaneous death compared to WT neutrophils, with $40 \%$ more 
healthy neutrophils in the GSDMD-deficient neutrophil population compared to WT (Figures 3E and S3C). Similar results were observed in peripheral blood neutrophils (Figure $3 \mathrm{~F})$. In the presence of E. coli, neutrophils die much faster.

We also examined the role of GSDMD in E. coli-induced neutrophil death, a process mediated mainly by phagocytosis. Again, we observed significantly delayed death in the GSDMD-deficient neutrophil population (Figures 3G, 3H, and S3D). Finally, we used an adoptive transfer assay to investigate whether GSDMD is involved intrinsically in neutrophil death in vivo (Figures 3I and 3J). In this assay, fluorescent-labeled WT and GSDMDdeficient neutrophils were injected directly into the peritoneal cavity of the same recipient mice so that we were able to assess the survival and death of WT and GSDMD-deficient neutrophils in the same environment. Consistent with the ex vivo data, significantly more GSDMD-deficient neutrophils were detected in the inflamed peritoneal cavity compared to WT neutrophils $15 \mathrm{hr}$ after the adoptive transfer (Figures 3I and 3J). The results of this experiment directly confirm that GSDMD disruption in neutrophils can intrinsically lead to delayed neutrophil death in vivo at the site of inflammation.

We also investigated whether GSDMD plays a role in neutrophil recruitment. Fluorescentlabeled WT and GSDMD-deficient neutrophils were injected into an TG-challenged WT receipt intravenously. Peritoneal neutrophil number was assessed at earlier time points when neutrophil death had not yet occurred. The ratio of WT to GSDMD-deficient neutrophils in the inflamed peritoneal cavity remained unaltered compared to the input (1:1), indicating that disruption of GSDMD in neutrophils did not affect neutrophil recruitment during infection and inflammation (Figures S3E and S3F).

\section{GSDMD Cleavage and Activation in Neutrophils Is Mediated by ELANE in a Caspase- Independent Manner}

Our results suggest that GSDMD may play a role in regulating neutrophil death. To further explore this hypothesis, we first investigated GSDMD expression in neutrophils. Examination of two publicly available transcriptomes of hematopoietic cells revealed that GSDMD is highly expressed in neutrophils at levels comparable to those in macrophages (Figures S4A and S4B). Consistent with this, GSDMD protein was present in both highly purified neutrophils and neutrophil-like differentiated HL60 cells (Figures 4A and 4B).

During macrophage pyroptosis, caspases cleave full-length GSDMD. To explore GSDMD cleavage activity in neutrophils, we incubated recombinant FLAG-tagged full-length GSDMD (FLAG-FL-GSDMD) with neutrophil lysate, thereby allowing us to track the cleaved NT GSDMD fragments. GSDMD could be processed by both human (Figure 4C) and mouse (Figure 4D) neutrophil lysates to generate an NT fragment of similar size to GSDMD-cNT. To investigate whether cleavage was caspase dependent, we included Z-TyrVal-Ala-Asp(OMe)-fluoromethyl-ketone (z-YVAD-fmk), a caspase inhibitor, in the cleavage reaction. z-YVAD-fmk efficiently blocked caspase-elicited GSDMD cleavage as expected (Loison et al., 2014), but it failed to suppress human (Figure 4E) or mouse (Figure 4F) neutrophil lysate-elicited GSDMD cleavage, suggesting that GSDMD cleavage by neutrophil lysate was caspase independent. Noticeably, human neutrophil lysate degraded all of the GSDMD protein species. Compared to the input, there was virtually no human 
GSDMD-NT (hGSDMD-NT) left after $30 \mathrm{~min}$. Instead, the mouse lysate generated a stable mouse GSDMD-NT (mGSDMD-NT) fragment. The rapid degradation of hGSDMD-NT could be caused by other proteases in human neutrophil lysate. We wondered whether GSDMD was cleaved by neutrophil serine proteases to generate an active GSDMD-NT fragment. To test this, neutrophil lysates were treated with diisopropylfluorophosphate (DFP), a potent and irreversible serine protease inhibitor. DFP treatment significantly reduced both human and mouse neutrophil lysate-induced GSDMD cleavage (Figures 4E and 4F). Thus, GSDMD cleavage and activation in neutrophils is caspase independent and is likely to be mediated by a serine protease.

To determine which serine protease may be responsible for GSDMD cleavage and activation, we incubated FLAG-hGSDMD with caspase-1 and each of the three major neutrophil serine proteases: proteinase 3 (PR3), ELANE, and cathepsin G. As previously reported, caspase-1 cleaved FL-GSDMD to generate GSDMD-cNT. We were intrigued that one of the neutrophil serine proteases, ELANE, also cleaved GSDMD to generate a GSDMD-NT fragment (GSDMD-eNT) that was slightly smaller than GSDMD-cNT. In contrast, PR3 and cathepsin $\mathrm{G}$ failed to produce a significant number, if any, of GSDMD-NT fragments (Figure 4G). Several cleaved bands were detected by an antibody recognizing GSDMD, perhaps representing non-specific and non-functional cleavage in the middle of GSDMD (Figure S4C). The degree of GSDMD cleavage was positively correlated with ELANE enzymatic activity in the reaction (Figure S4D), and the cleavage was efficiently inhibited by ELANE-specific inhibitors sivelestat (Figure S4E), BAY-678 (Figure S4F), and GW311616A (Figure S4G) and by the pan-serine protease inhibitor DFP (Figure S4H). More important, GSDMD cleavage by whole human neutrophil lysate also was reduced significantly by ELANE-specific inhibitors in a dose-dependent manner (Figure 4H), and the GSDMD-NT fragment produced by neutrophil lysate was the same size as the one cleaved by ELANE (Figures S4I and S4J). Therefore, GSDMD cleavage activity in human neutrophil lysate appears to be attributable mainly to ELANE.

We next sought to investigate whether ELANE also mediates mGSDMD cleavage. Active recombinant mouse ELANE is not commercially available, so we incubated ELANE precursor with active cathepsin C/dipeptidylpeptidase I (DPPI), which removes dipeptide from the ELANE precursor to generate active mature ELANE (Adkison et al., 2002). In parallel to ELANE activation, mGSDMD was processed into two major NT fragments, one of which was a similar size to that of GSDMD-cNT (Figure 4I). ELANE-specific protease inhibitors inhibited generation of this fragment in a dose-dependent manner (Figure S4K), as did ELANE-deficient neutrophil lysate (Figure 4J). Thus, both mouse and human ELANE can effectively and efficiently cleave GSDMD to produce GSDMD-eNT in neutrophils. As a control, caspase-1/11 double KO (DKO) neutrophil lysate cleaved mGSDMD as efficiently as the WT neutrophil lysate, further indicating that GSDMD cleavage in neutrophils is caspase-1/11-independent (Figure 4K). Caspase-1/11 DKO mice consistently did not exhibit significantly elevated neutrophil accumulation in the peritonitis model, again confirming that the elevated neutrophil accumulation observed in the GSDMD-deficient KO is caspase-1/11 independent (Figure 4L). 


\section{Lysosomal Membrane Permeabilization Induces ELANE Release into the Cytosol, Leading to GSDMD Cleavage}

GSDMD must co-localize with ELANE in neutrophils for cleavage, so we next examined this activity. In healthy neutrophils, the cytoplasmic granules mediate antimicrobial activity and contain all three serine proteinases, with the granule contents released during neutrophil death via lysosomal membrane permeabilization (LMP) (Figure 5A) (Loison et al., 2014). LMP was triggered in both spontaneous and E. coli-induced neutrophil death (Figures 5A and 5B). Cytosolic ELANE was consistently low in fresh healthy neutrophils and increased significantly during neutrophil death (Figures 5C and 5D), supporting a model in which LMP-induced ELANE release into the cytosol leads to GSDMD cleavage to facilitate neutrophil death. Indeed, we previously showed that the inhibition of LMP drastically delays constitutive neutrophil death (Loison et al., 2014). Consistent with our hypothesis, treatment with ELANE-specific inhibitor delayed significantly the death program in human neutrophils (Figure 5E). In addition, treatment with ELANE inhibitor could further promote the survival of neutrophils treated with the caspase-1/4/5-specific inhibitor, indicating that the effect of ELANE is independent of caspase-mediated GSDMD cleavage. Thus, ELANE appears to be a key regulatory protease that cleaves GSDMD to produce GSDMD-eNT during neutrophil death. Similar to what was observed in spontaneous neutrophil death, treatment with ELANE inhibitor also could promote the survival of cultured human neutrophils in the presence of E. coli infection (Figure 5F). It is noteworthy that ELANE inhibitor-induced suppression of neutrophil death was not as prominent as the one elicited by GSDMD disruption, indicating that ELANE may not be the only protease that cleaves and activates GSDMD in neutrophils. We next examined the bacterial killing capability of the untreated and ELANE inhibitor-treated neutrophil cultures (Figure 5G). ELANE inhibitor-induced delayed death of cultured human neutrophils led to augmented bacterial killing, consistent with the increased healthy neutrophil number in these cultures. We next assessed the neutrophil chemotaxis capability using a EZ-TAXIScan (ECI Frontier Inc) device (Hattori et al., 2010). It is interesting that the chemotaxis and phagocytosis capabilities of the surviving cells were unaltered (Figures S5A and S5B). This is consistent with the well-accepted mechanism by which GSDMD mediates cell death. The transiently generated GSDMD-NT triggers neutrophil death immediately upon its generation, leading to the attenuated overall bacterial killing capability of neutrophil culture. However, the surviving cells likely do not contain high levels of GSDMD-NT and thus function normally.

\section{ELANE Cleaves GSDMD Upstream of the Caspase Cleavage Site to Generate a Smaller but Still Biologically Active GSDMD-eNT Fragment}

GSDMD-eNT is slightly smaller than GSDMD-cNT on western blots. To determine the exact ELANE cutting site on GSDMD, we produced both human and mouse recombinant His-SUMO-GSDMD in E. coli, using the NT GSDMD-His-SUMO tag fusion to specifically purify GSDMD-eNT fragments after cleavage for subsequent mass spectrometry (MS) (Figure 6A). hGSDMD was incubated with ELANE or human neutrophil lysate, and intact and cleaved GSDMD bands were excised and subjected to liquid chromatography-MS (Figure 6B). Analysis of trypsin-digested fragments derived from FL-hGSDMD and hGSDMD-eNT localized the cleavage site to a seven-amino acid sequence between 268 and 274 (Figure 6C). We then generated a series of hGSDMD deletion mutants between amino 
acids 247 and 282 . GSDMD- $\Delta 268-274$ and $-\Delta 268-270$ failed to be cleaved efficiently by ELANE, suggesting that the cleavage site localizes between C268 and H270 (Figure 6D). Because glycine is found rarely at ELANE cleavage sites and is thought to be an amino acid that cannot be cleaved by ELANE (Camper et al., 2016), we made glycine point mutants at 268, 269, and 270. GSDMD-C268G but not other GSDMD mutants generated significantly less GSDMD-eNT in response to treatment with ELANE (Figure 6E), pinpointing the ELANE cleavage site on hGSDMD to C268 and further confirming by MS analysis AspNdigested hGSDMD fragments. A peptide ending at C268 was identified in the GSDMD-eNT sample but not in the sample containing FL-hGSDMD (Figures 6F and 6G). The identified ELANE cleavage site, C268, was seven amino acids upstream of the caspase cleavage site D275 (Figure 6H). Using a similar approach, we revealed that mGSDMD was cleaved at V251 (Figures S6A-S6F). Notably, the GSDMD ELANE cleavage site was not well conserved between humans and mice (Figure S6G), suggesting that ELANE may recognize a tertiary structure rather than a particular amino acid sequence in GSDMD. These results are consistent with the known preference of ELANE for small hydrophobic amino acids such as Val, Cys, and Ala at the P1 position of protein substrates (Korkmaz et al., 2008).

The ELANE and caspase cleavage sites in GSDMD are, therefore, different. We next examined whether ELANE-mediated GSDMD cleavage also activates it by expressing human GSDMD-eNT (hGSDMD 1-268) and GSDMD-cNT (hGSDMD 1-275) in 293T cells (Figure 7A). GSDMD-NT-mediated plasma membrane pore formation depends on the oligomerization of GSDMD-NT molecules. Both hGSDMD-eNT and hGSDMD-cNT efficiently formed oligomers (Figure 7B) and localized to the plasma membrane (Figure 7C). Both hGSDMD-eNT and hGSDMD-cNT but not the FL or hGSDMD1 to 225, a truncated inactive form of hGSDMD, consistently induced lytic death of 293T cells (Figure 7D). The dying cells displayed typical lytic morphology of cell swelling and rupture and leakage of the plasma membrane, leading to intracellular DNA staining by membrane-impermeable PI (Movies S1, S2, S3, and S4). In addition, death-associated release of lactate dehydrogenase (LDH) also was comparable between hGSDMD-eNT- and hGSDMD-cNT-expressing cells (Figure 7E). Similar cytotoxicity also was observed when mGSDMD-eNTs (mGSDMD1251) were expressed in $293 \mathrm{~T}$ cells (Figures S7A and S7B). Collectively, these results show that ELANE-cleaved GSDMD is biologically active and induces lytic cell death, which are consistent with previous findings that the minimum length of active GSDMD-NT is $\sim 243$ amino acids (Shi et al., 2015a).

\section{DISCUSSION}

GSDM family proteins are inactive in their steady state because of autoinhibition by the Cterminus domain (He et al., 2015; Shi et al., 2015a; Shi et al., 2015b). Their activation relies on cleavage of the FL protein to generate the NT active fragment. GSDM cleavage may be context dependent and mediated by distinct mechanisms in different tissues and cells.

ELANE-meditated GSDMD cleavage is likely to be a specific GSDMD activation mechanism in neutrophils because other cell types, including monocyte-derived macrophages (MDMs), do not express ELANE (Figures S7C and S7D). Alternatively, ELANE may not be the only protease that cleaves and activates GSDMD in neutrophils. An NT fragment slightly larger than GSDMD-eNT was still generated in the absence of ELANE 
and also may participate in lytic cell death (Figure 4J). Inflammasome-mediated caspase-1 activation has been implicated in neutrophil-mediated cytokine release and neutrophilic inflammation (Bakele et al., 2014; Chen et al., 2014; Karmakar et al., 2016), and thus may elicit GSDMD cleavage and activation in neutrophils independent of ELANE. Nevertheless, caspase-1/11 DKO neutrophil lysate cleaved mGSDMD as efficiently as the WT neutrophil lysate to generate mGSDMD-NT (Figure 4K). In addition, caspase-1/11 DKO mice did not exhibit elevated neutrophil accumulation in the peritonitis model, confirming that the elevated neutrophil accumulation observed in the GSDMD-deficient KO is caspase independent.

In macrophages, cleavage of GSDMD induces membrane pore formation and proinflammatory cytokine (e.g., IL-1 $\beta$ ) secretion (Aglietti et al., 2016; Chen et al., 2016; Ding et al., 2016; He et al., 2015; Liu et al., 2016; Sborgi et al., 2016). GSDMD-cNT also binds to cardiolipin in the inner and outer leaflets of bacterial membranes, suggesting that it may directly kill bacteria by perforating bacterial membranes (Ding et al., 2016; Liu et al., 2016). On the basis of these studies, GSDMD is generally considered proinflammatory and an important host response to bacterial infection (Kayagaki et al., 2015; Shi et al., 2015a). Here, we reveal that GSDMD deficiency unexpectedly and paradoxically augments host defense against extracellular E. coli by delaying neutrophil death. GSDMD controls neutrophil death and negatively regulates neutrophil-mediated innate immunity. Because the dead neutrophils are rapidly engulfed and cleared by macrophages, this process generally reduces neutrophil number and is mainly anti-inflammatory. The levels of proinflammatory cytokines in the peritoneal cavity were elevated significantly in GSDMD-deficient mice, suggesting that the production of these cytokines was not mediated mainly by GSDMDmediated macrophage pyroptosis. This further confirmed that in E. coli-induced peritonitis, GSDMD acted as an anti-inflammatory factor. Thus, GSDMD can exert context-dependent proinflammatory and anti-inflammatory effects, making it a unique target for antibacterial and anti-inflammatory therapies (Figure S7E). Although both our in vitro and in vivo data demonstrated directly that GSDMD in neutrophils can intrinsically regulate neutrophil death during infection and inflammation, we cannot completely exclude the contribution of other cell types in the regulation of neutrophil accumulation in GSDMD-deficient mice. It also is noteworthy that the effect of GSDMD disruption on neutrophil death was not as drastic as its effect on in vivo bacteria killing. It is likely that a modest difference in neutrophil death can upregulate inflammation, leading to more neutrophil recruitment and a log difference in bacterial clearance.

Our results support a model in which ELANE cleaves and activates GSDMD to perforate the plasma membrane and induce lytic cell death in neutrophils. It is noteworthy that depletion of GSDMD did not completely block lytic neutrophil death, suggesting that other parallel pathways exist to facilitate neutrophil death. In fact, the canonical caspase-3-mediated mechanism is well documented to regulate neutrophil death. Alternatively, other GSDM family proteins may be expressed in neutrophils and cleaved by neutrophil proteases to promote neutrophil death in the absence of GSDMD. Apoptosis has long been considered the primary mechanism of cell death for aging neutrophils, but inhibition of caspases, which are critical mediators of apoptosis, delay but do not completely abolish cell death, suggesting that other mechanisms of neutrophil death exist (Loison et al., 2014; Luo and 
Loison, 2008). Our most recent study showed that neutrophil death consists of not only apoptosis but also a heterogeneous collection of different types of cell death (Kasorn et al., 2009; Teng et al., 2017). Here, we show that GSDMD-mediated lytic cell death also contributes to neutrophil death.

Serine proteases, including ELANE, are stored mainly in azurophilic granules at concentrations exceeding $5 \mathrm{mM}$ (Borregaard and Cowland, 1997). They are key components of the innate immune system and mediate pathogen clearance via multiple mechanisms, including direct suppression of pathogen proliferation and survival, production of antimicrobial peptides, deactivation of virulence factors, and generation of neutrophil extracellular traps (NETs) (Stapels et al., 2015; Tkalcevic et al., 2000). ELANE-mediated degradation of bacterial outer membrane protein A (OmpA) can kill bacteria directly (Belaaouaj et al., 2000). ELANE-deficient mice displayed impaired host defenses against Gram-negative bacterial sepsis (Belaaouaj et al., 1998). It is interesting that mice lacking both PR3 and ELANE have been linked to accumulation of anti-inflammatory progranulin (PGRN) and exaggerated inflammation, suggesting a cooperative interaction between serine proteases (Kessenbrock et al., 2008). Here, we discovered an alternative mechanism by which ELANE regulates innate immunity by promoting neutrophil death.

The present study further demonstrates the significance of LMP in promoting neutrophil death (Figure S7F). We previously showed that another serine protease, PR3, is released from granules into the cytosol via LMP in aging neutrophils to cleave and activate caspase-3 to cause neutrophil death (Loison et al., 2014). Inhibition of LMP significantly curtails PR3 release from neutrophil granules and consequently delays neutrophil death. Here, we show that ELANE and ELANE-mediated GSDMD cleavage are other key mechanisms that mediate LMP-elicited neutrophil death. Thus, LMP should be a legitimate target for regulating neutrophil death in infection and inflammation. The mechanism leading to LMP is still ill-defined. Reactive oxygen species (ROS) accumulate in aging neutrophils and mediate neutrophil death (Xu et al., 2013; Xu et al., 2010). ROS-induced lipid peroxidation may directly cause rupture of granule membranes and LMP. Electron microscopy examination reveals obvious granule membrane rupture in aging neutrophils (Loison et al., 2014). A recent study showed that ELANE release during NETosis also is triggered by ROS (Metzler et al., 2014). In resting neutrophils, ELANE is localized on the azurophilic granule membrane and associated with a multiprotein complex known as azurosome. Upon stimulation, oxidative burst generates ROS that in turn triggers dissociation of ELANE from azurosome into the cytosol and the subsequent translocation to the nucleus. It would be intriguing to investigate whether this azurosome-mediated mechanism also plays a role in neutrophil death and whether the azurosome-mediated pathway and LMP can regulate each other.

\section{EXPERIMENTAL PROCEDURES}

\section{Mice}

Eight- to 12-week-old male mice were used in all of the experiments. Corresponding littermates were used as WT controls in all of the experiments performed with KO mice. The 
Boston Children's Hospital Animal Care and Use Committee approved and monitored all of the procedures involving mice.

\section{Neutrophil Apoptosis in the Inflamed Peritoneal Cavity}

Peritonitis was induced by intraperitoneally injection with $5 \times 10^{7}$ E. coli (strain K12 BW25113) in PBS. At indicated times after injection, the mice were euthanized with $\mathrm{CO}_{2}$. Peritoneal exudate cells were then harvested by three successive washes with $10 \mathrm{~mL}$ Hank's balanced salt solution (HBSS) containing $0.2 \%$ BSA and $20 \mathrm{mM}$ EDTA. The cells were stained with annexin V (fluorescein isothiocyanate [FITC] labeled, Invitrogen) and PI, and cell death was then analyzed by FACS using a FACSCanto II flow cytometer (Becton Dickinson, San Jose, CA).

\section{Statistics}

Analysis of statistical significance for the indicated datasets was performed with the twotailed Student's t test using GraphPad Prism 5 (GraphPad Software, La Jolla, CA). p values $<0.05$ were considered significant.

\section{Supplementary Material}

Refer to Web version on PubMed Central for supplementary material.

\section{Acknowledgments}

The authors thank John Manis, Feng Shao, and Li Chai for helpful discussions, and Harry Leung for technical assistance with confocal microscopy. Y.X. is supported by grants from the National Basic Research Program of China (2015CB964903), the Chinese Academy of Medical Sciences (CAMS) Innovation Fund for Medical Sciences (2016-12M-1-003 and 2017-I2M-1-015), and the Chinese National Natural Science Foundation (31471116). F.L. is supported by a grant from the Chinese National Natural Science Foundation (31700783), the Peking Union Medical College (PUMC) Youth Fund, and the Fundamental Research Funds for the Central Universities (2017310023). H.L. is supported by NIH grants (R01AI103142, R01HL092020, and P01HL095489) and a grant from the Flight Attendant Medical Research Institute (FAMRI) (CIA 123008). pCSII-EF-RfA and pCSII-CMV-MCS-IRES2-Bsd vectors were kindly supplied by Dr. Hiroyuki Miyoshi (RIKEN BioResource Center). The pCAG-MCS2-FOS vector was kindly supplied by Dr. Eiji Morita (Hirosaki University).

\section{References}

Adkison AM, Raptis SZ, Kelley DG, Pham CT. Dipeptidyl peptidase I activates neutrophil-derived serine proteases and regulates the development of acute experimental arthritis. J Clin Invest. 2002; 109:363-371. [PubMed: 11827996]

Aglietti RA, Estevez A, Gupta A, Ramirez MG, Liu PS, Kayagaki N, Ciferri C, Dixit VM, Dueber EC. GsdmD p30 elicited by caspase-11 during pyroptosis forms pores in membranes. Proc Natl Acad Sci USA. 2016; 113:7858-7863. [PubMed: 27339137]

Bakele M, Joos M, Burdi S, Allgaier N, Pöschel S, Fehrenbacher B, Schaller M, Marcos V, KümmerleDeschner J, Rieber N, et al. Localization and functionality of the inflammasome in neutrophils. J Biol Chem. 2014; 289:5320-5329. [PubMed: 24398679]

Belaaouaj A, McCarthy R, Baumann M, Gao Z, Ley TJ, Abraham SN, Shapiro SD. Mice lacking neutrophil elastase reveal impaired host defense against gram negative bacterial sepsis. Nat Med. 1998; 4:615-618. [PubMed: 9585238]

Belaaouaj A, Kim KS, Shapiro SD. Degradation of outer membrane protein A in Escherichia coli killing by neutrophil elastase. Science. 2000; 289:1185-1188. [PubMed: 10947984]

Borregaard N, Cowland JB. Granules of the human neutrophilic polymorphonuclear leukocyte. Blood. 1997; 89:3503-3521. [PubMed: 9160655] 
Camper N, Glasgow AM, Osbourn M, Quinn DJ, Small DM, McLean DT, Lundy FT, Elborn JS, McNally P, Ingram RJ, et al. A secretory leukocyte protease inhibitor variant with improved activity against lung infection. Mucosal Immunol. 2016; 9:669-676. [PubMed: 26376365]

Chen KW, Groß CJ, Sotomayor FV, Stacey KJ, Tschopp J, Sweet MJ, Schroder K. The neutrophil NLRC4 inflammasome selectively promotes IL-1 $\beta$ maturation without pyroptosis during acute Salmonella challenge. Cell Rep. 2014; 8:570-582. [PubMed: 25043180]

Chen X, He WT, Hu L, Li J, Fang Y, Wang X, Xu X, Wang Z, Huang K, Han J. Pyroptosis is driven by non-selective gasdermin-D pore and its morphology is different from MLKL channel-mediated necroptosis. Cell Res. 2016; 26:1007-1020. [PubMed: 27573174]

Ding J, Wang K, Liu W, She Y, Sun Q, Shi J, Sun H, Wang DC, Shao F. Pore-forming activity and structural autoinhibition of the gasdermin family. Nature. 2016; 535:111-116. [PubMed: 27281216]

Hattori H, Subramanian KK, Sakai J, Jia Y, Li Y, Porter TF, Loison F, Sarraj B, Kasorn A, Jo H, et al. Small-molecule screen identifies reactive oxygen species as key regulators of neutrophil chemotaxis. Proc Natl Acad Sci USA. 2010; 107:3546-3551. [PubMed: 20142487]

He WT, Wan H, Hu L, Chen P, Wang X, Huang Z, Yang ZH, Zhong CQ, Han J. Gasdermin D is an executor of pyroptosis and required for interleukin-1 $\beta$ secretion. Cell Res. 2015 ; 25:1285-1298. [PubMed: 26611636]

Jorgensen I, Miao EA. Pyroptotic cell death defends against intracellular pathogens. Immunol Rev. 2015; 265:130-142. [PubMed: 25879289]

Jorgensen I, Zhang Y, Krantz BA, Miao EA. Pyroptosis triggers pore-induced intracellular traps (PITs) that capture bacteria and lead to their clearance by efferocytosis. J Exp Med. 2016; 213:21132128. [PubMed: 27573815]

Karmakar M, Katsnelson MA, Dubyak GR, Pearlman E. Neutrophil P2X7 receptors mediate NLRP3 inflammasome-dependent IL-1 $\beta$ secretion in response to ATP. Nat Commun. 2016; 7:10555. [PubMed: 26877061]

Kasorn A, Alcaide P, Jia Y, Subramanian KK, Sarraj B, Li Y, Loison F, Hattori H, Silberstein LE, Luscinskas WF, Luo HR. Focal adhesion kinase regulates pathogen-killing capability and life span of neutrophils via mediating both adhesion-dependent and -independent cellular signals. J Immunol. 2009; 183:1032-1043. [PubMed: 19561112]

Kayagaki N, Stowe IB, Lee BL, O’Rourke K, Anderson K, Warming S, Cuellar T, Haley B, RooseGirma M, Phung QT, et al. Caspase-11 cleaves gasdermin D for non-canonical inflammasome signalling. Nature. 2015; 526:666-671. [PubMed: 26375259]

Kessenbrock K, Fröhlich L, Sixt M, Lämmermann T, Pfister H, Bateman A, Belaaouaj A, Ring J, Ollert M, Fässler R, Jenne DE. Proteinase 3 and neutrophil elastase enhance inflammation in mice by inactivating antiinflammatory progranulin. J Clin Invest. 2008; 118:2438-2447. [PubMed: 18568075]

Korkmaz B, Moreau T, Gauthier F. Neutrophil elastase, proteinase 3 and cathepsin G: physicochemical properties, activity and physiopathological functions. Biochimie. 2008; 90:227-242. [PubMed: 18021746]

Lahoz-Beneytez J, Elemans M, Zhang Y, Ahmed R, Salam A, Block M, Niederalt C, Asquith B, Macallan D. Human neutrophil kinetics: modeling of stable isotope labeling data supports short blood neutrophil half-lives. Blood. 2016; 127:3431-3438. [PubMed: 27136946]

Li Y, Jia Y, Pichavant M, Loison F, Sarraj B, Kasorn A, You J, Robson BE, Umetsu DT, Mizgerd JP, et al. Targeted deletion of tumor suppressor PTEN augments neutrophil function and enhances host defense in neutropenia-associated pneumonia. Blood. 2009; 113:4930-4941. [PubMed: 19286998]

Li Y, Prasad A, Jia Y, Roy SG, Loison F, Mondal S, Kocjan P, Silberstein LE, Ding S, Luo HR. Pretreatment with phosphatase and tensin homolog deleted on chromosome 10 (PTEN) inhibitor SF1670 augments the efficacy of granulocyte transfusion in a clinically relevant mouse model. Blood. 2011; 117:6702-6713. [PubMed: 21521784]

Liu X, Zhang Z, Ruan J, Pan Y, Magupalli VG, Wu H, Lieberman J. Inflammasome-activated gasdermin D causes pyroptosis by forming membrane pores. Nature. 2016; 535:153-158. [PubMed: 27383986] 
Loison F, Zhu H, Karatepe K, Kasorn A, Liu P, Ye K, Zhou J, Cao S, Gong H, Jenne DE, et al. Proteinase 3-dependent caspase-3 cleavage modulates neutrophil death and inflammation. J Clin Invest. 2014; 124:4445-4458. [PubMed: 25180606]

Lord BI, Woolford LB, Molineux G. Kinetics of neutrophil production in normal and neutropenic animals during the response to filgrastim (r-metHu G-CSF) or filgrastim SD/01 (PEG-r-metHu GCSF). Clin Cancer Res. 2001; 7:2085-2090. [PubMed: 11448927]

Luo HR, Loison F. Constitutive neutrophil apoptosis: mechanisms and regulation. Am J Hematol. 2008; 83:288-295. [PubMed: 17924549]

Metzler KD, Goosmann C, Lubojemska A, Zychlinsky A, Papayannopoulos V. A myeloperoxidasecontaining complex regulates neutrophil elastase release and actin dynamics during NETosis. Cell Rep. 2014; 8:883-896. [PubMed: 25066128]

Poon IK, Lucas CD, Rossi AG, Ravichandran KS. Apoptotic cell clearance: basic biology and therapeutic potential. Nat Rev Immunol. 2014; 14:166-180. [PubMed: 24481336]

Savill JS, Wyllie AH, Henson JE, Walport MJ, Henson PM, Haslett C. Macrophage phagocytosis of aging neutrophils in inflammation. Programmed cell death in the neutrophil leads to its recognition by macrophages. J Clin Invest. 1989; 83:865-875. [PubMed: 2921324]

Sborgi L, Rühl S, Mulvihill E, Pipercevic J, Heilig R, Stahlberg H, Farady CJ, Müller DJ, Broz P, Hiller S. GSDMD membrane pore formation constitutes the mechanism of pyroptotic cell death. EMBO J. 2016; 35:1766-1778. [PubMed: 27418190]

Shi J, Zhao Y, Wang K, Shi X, Wang Y, Huang H, Zhuang Y, Cai T, Wang F, Shao F. Cleavage of GSDMD by inflammatory caspases determines pyroptotic cell death. Nature. 2015a; 526:660-665. [PubMed: 26375003]

Shi P, Tang A, Xian L, Hou S, Zou D, Lv Y, Huang Z, Wang Q, Song A, Lin Z, Gao X. Loss of conserved Gsdma3 self-regulation causes autophagy and cell death. Biochem J. 2015b; 468:325336. [PubMed: 25825937]

Stapels DA, Geisbrecht BV, Rooijakkers SH. Neutrophil serine proteases in antibacterial defense. Curr Opin Microbiol. 2015; 23:42-48. [PubMed: 25461571]

Teng Y, Luo HR, Kambara H. Heterogeneity of neutrophil spontaneous death. Am J Hematol. 2017; 92:E156-E159. [PubMed: 28437875]

Tkalcevic J, Novelli M, Phylactides M, Iredale JP, Segal AW, Roes J. Impaired immunity and enhanced resistance to endotoxin in the absence of neutrophil elastase and cathepsin G. Immunity. 2000; 12:201-210. [PubMed: 10714686]

$\mathrm{Xu}$ Y, Loison F, Luo HR. Neutrophil spontaneous death is mediated by down-regulation of autocrine signaling through GPCR, PI3Kgamma, ROS, and actin. Proc Natl Acad Sci USA. 2010; 107:2950-2955. [PubMed: 20133633]

Xu Y, Li H, Bajrami B, Kwak H, Cao S, Liu P, Zhou J, Zhou Y, Zhu H, Ye K, Luo HR. Cigarette smoke (CS) and nicotine delay neutrophil spontaneous death via suppressing production of diphosphoinositol pentakisphosphate. Proc Natl Acad Sci USA. 2013; 110:7726-7731. [PubMed: 23610437] 


\section{Highlights}

- GSDMD-deficient mice display augmented bactericidal activity against Escherichia coli

- GSDMD is a key regulator of neutrophil death

- Delayed neutrophil death leads to enhanced host defense in GSDMDdeficient mice

- GSDMD cleavage in neutrophils is mediated by neutrophil elastase 
A

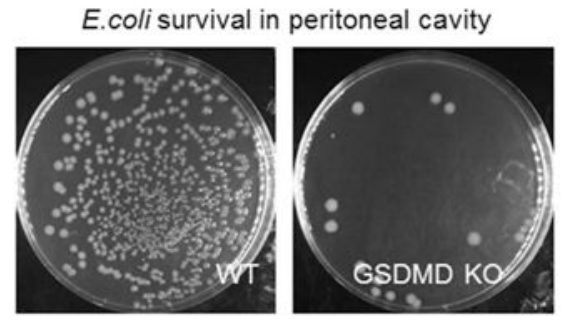

B
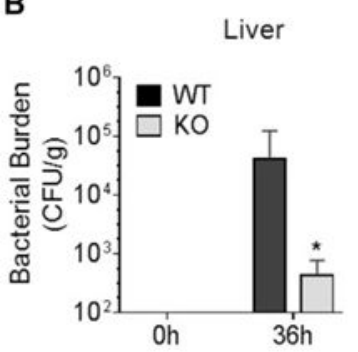

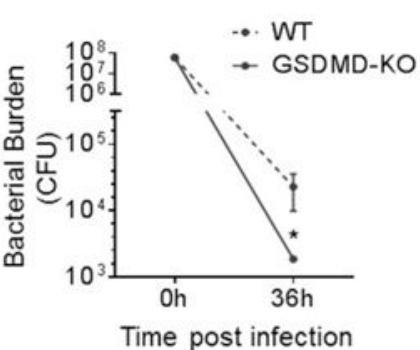

Spleen

Kidney
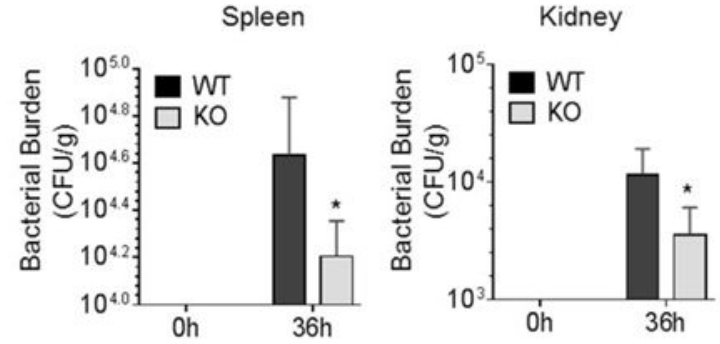

Figure 1. GSDMD-Deficient Mice Display Augmented Bactericidal Activity

(A) E. coli clearance in inflamed peritoneal cavities. Live bacteria were quantified as total colony-forming units (CFU) in peritoneal lavage fluid (PLF).

(B) Total CFUs in the indicated organs and peripheral blood $36 \mathrm{hr}$ after $E$. coli challenge. 
A

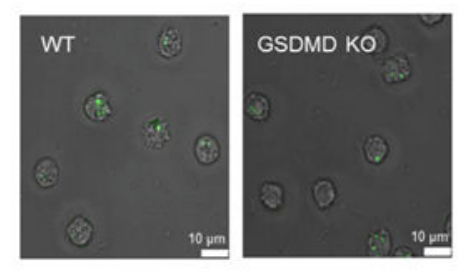

C

C PB neutrophils
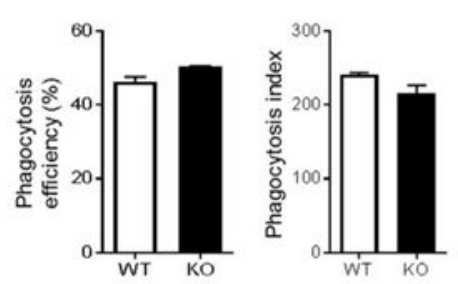

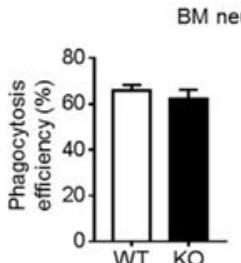

BM neutrophils

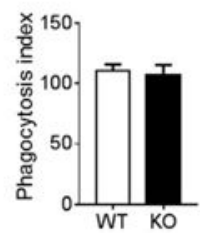

D
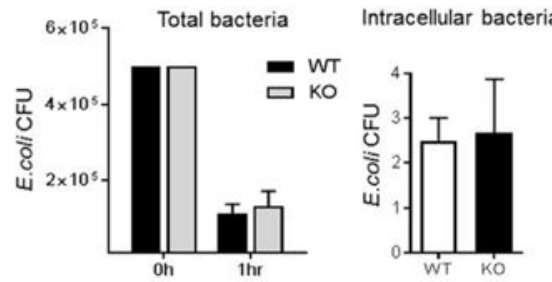

$\mathbf{F}$
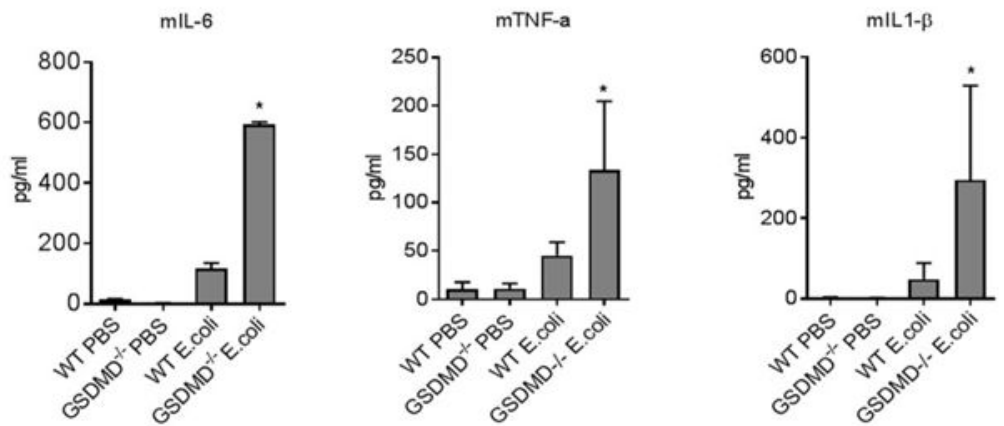

B

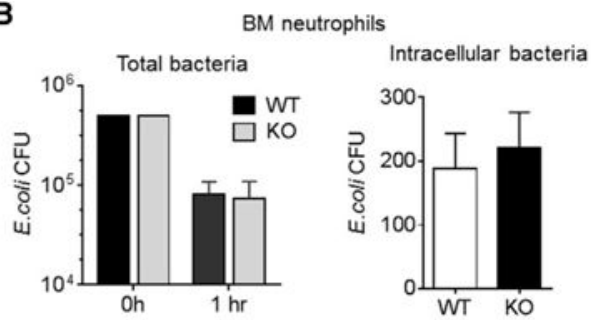

E
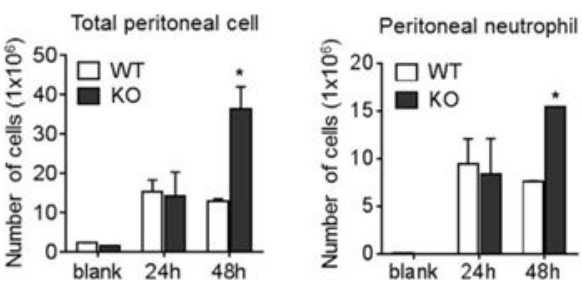

Figure 2. The Augmented Host Antibacterial Response in GSDMD-Deficient Mice Is the Result of Enhanced Neutrophil Accumulation

(A) In vitro phagocytosis assay. More than 200 neutrophils were counted in each group.

(B) In vitro killing of E. coli by bone marrow neutrophils. In vitro bacterial killing capabilities were reflected by the decrease in CFUs. Intracellular bactericidal activity of neutrophils was assessed by treating samples with kanamycin to kill extracellular bacteria.

(C) Phagocytosis of E. coli bioparticles by peripheral blood neutrophils. Results are the means $( \pm$ SDs $)$ of three independent experiments.

(D) In vitro killing of $E$. coli by peripheral blood neutrophils. Results are the means $( \pm$ SDs) of three independent experiments.

(E) Total peritoneal cell and peritoneal neutrophil numbers in E. coli-challenged mice. Data shown at each time point are means \pm SDs of four mice.

(F) Chemokine and cytokine levels in peritoneal cavity.

Results are the means $( \pm$ SDs) of three independent experiments. $* p<0.05$ versus WT. 
A
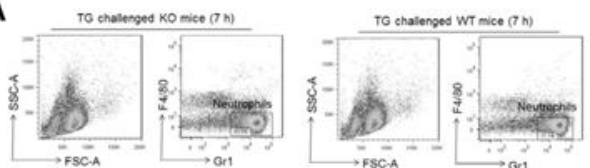

B
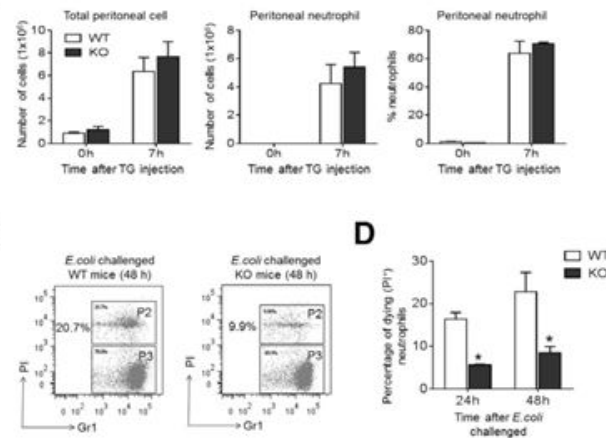

I

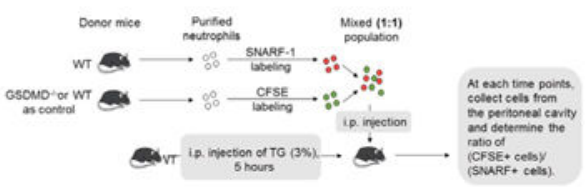

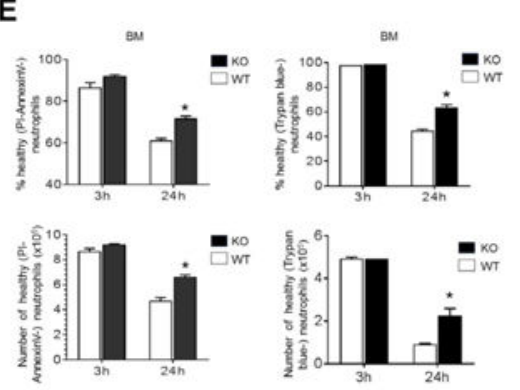

G
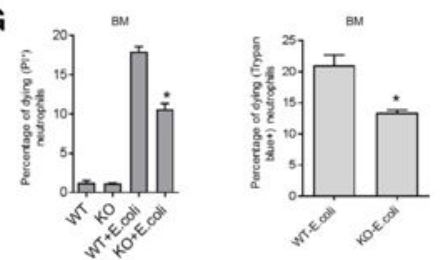

$J$

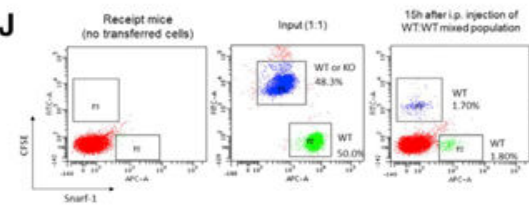

$\mathbf{F}$
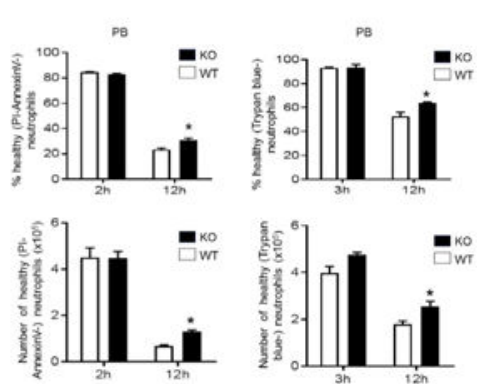

H
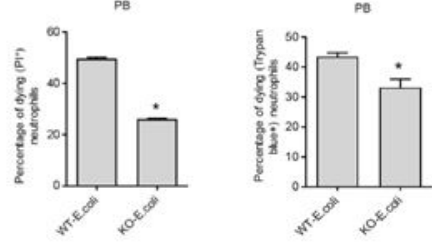

Figure 3. Delayed Neutrophil Death Elevates Neutrophil Accumulation and Enhances the Host Defense Response in GSDMD-Deficient Mice

(A) Neutrophil recruitment during sterile inflammation. At the indicated times, TGchallenged mice were killed and peritoneal lavage fluid collected for FACS. Shown are representative FACS plots depicting recruited peritoneal neutrophils.

(B) Total neutrophil content in the lavage fluid was calculated based on FACS analysis. Data shown at each time point are means \pm SDs of three mice.

(C) Neutrophil death during E. coli-induced peritonitis. Peritoneal cells were collected $48 \mathrm{hr}$ after E. coli injection and then analyzed by FACS. Shown are representative FACS plots depicting dying peritoneal neutrophils (P2). See also Figures S2D and S2E.

(D) The percentage of dying neutrophils in the lavage fluid was calculated based on FACS analysis. Data shown at each time point are means \pm SDs of three mice.

(E) Death of in vitro cultured WT and GSDMD-deficient bone marrow neutrophils. Shown are the percentage and absolute number of healthy neutrophils in the culture at time points indicated. Data shown are means \pm SDs of three mice. See also Figures S3A-S3D.

(F) Death of in vitro cultured WT and GSDMD-deficient peripheral blood neutrophils. All of the values represent means \pm SDs of three separate experiments.

(G) Death of in vitro cultured WT and GSDMD-deficient bone marrow neutrophils in the presence of E. coli. Bone marrow neutrophils were cultured with E. coli (1:5 ratio) for $2 \mathrm{hr}$. Data shown are means \pm SDs of three mice.

(H) Death of in vitro cultured WT and GSDMD-deficient peripheral blood neutrophils in the presence of $E$. coli. Data shown are means \pm SDs of three mice.

(I) In vivo death of adoptively transferred neutrophils. 
(J) The ratios of adoptively transferred WT and GSDMD-deficient neutrophils. The input control was normalized to 1 , and the results shown are the ratios at $15 \mathrm{hr}$ after the adoptive transfer.

All of the values represent means \pm SDs. ${ }^{*} \mathrm{p}<0.05$ versus WT. 
A

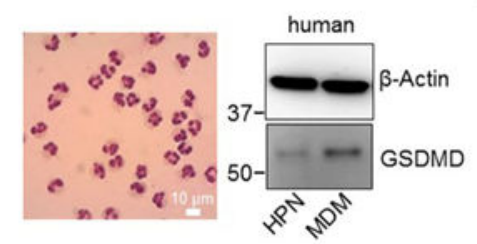

B

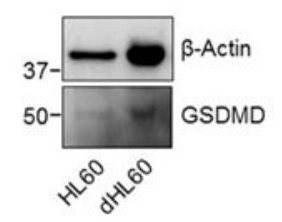

C

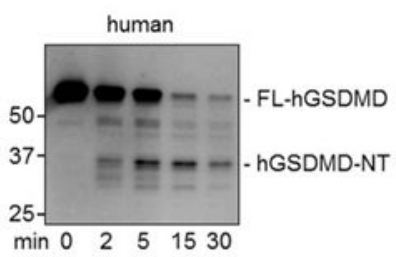

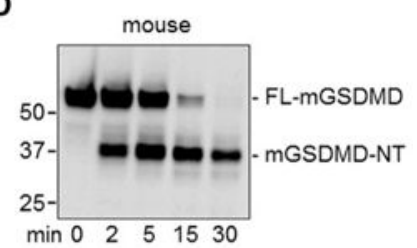

E

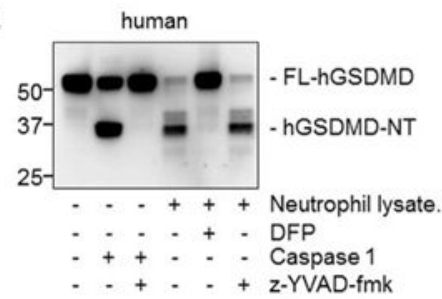

H
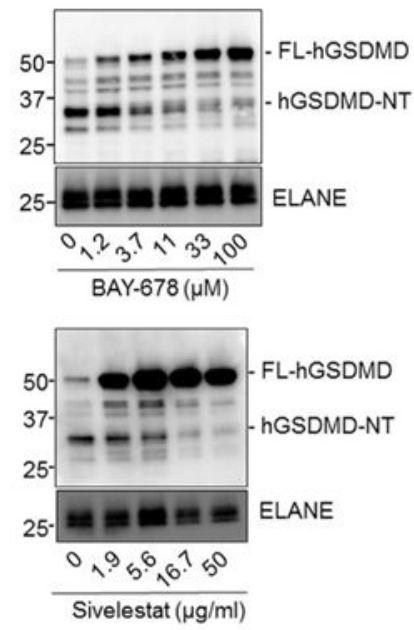

F
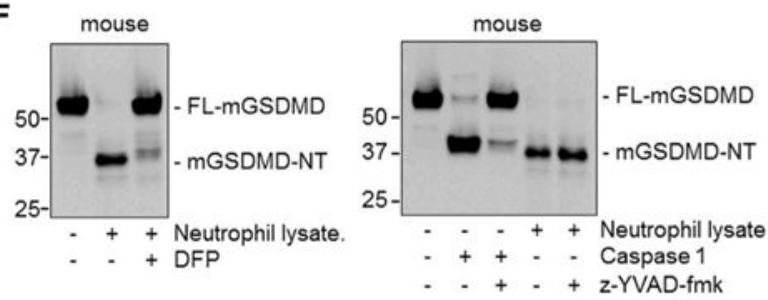

I

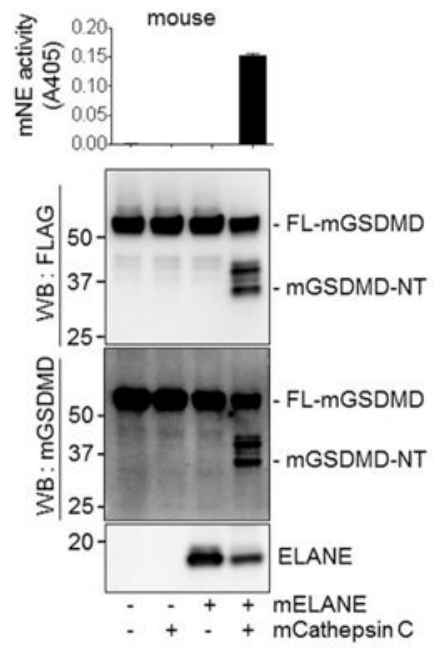

J

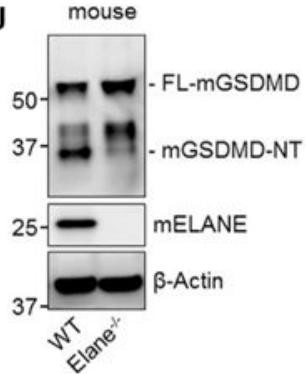

L

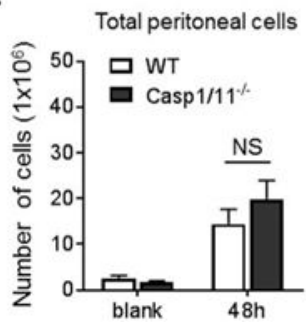

G

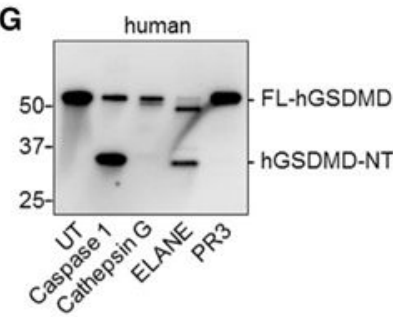

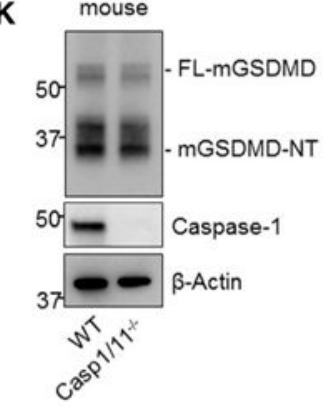

Peritoneal neutrophil

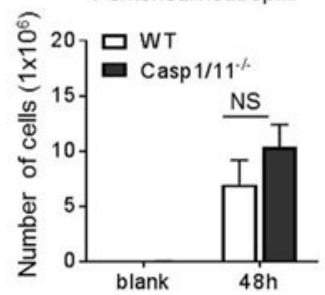

Figure 4. The Cleavage and Activation of GSDMD in Neutrophils Is Mediated by ELANE in a Caspase-Independent Manner

(A) GSDMD is highly expressed in neutrophils. GSDMD in human neutrophils and monocyte-derived macrophages (MDMs) was detected by western blotting with antiGSDMD antibody. The purity of highly purified neutrophils (HPNs) was confirmed by Wright-Giemsa staining of cytospin samples.

(B) GSDMD expression in undifferentiated and differentiated HL60 cells.

(C) Cleavage of human GSDMD (hGSDMD) by human neutrophil lysate. FLAG-hGSDMD was overexpressed in HEK293T cells. Cell lysates containing recombinant FLAG-hGSDMD were incubated with human neutrophil lysate for the times indicated. The positions of the full-length (FL-GSDMD) and ELANE-cleaved N-terminal hGSDMD (hGSDMD-NT) are indicated.

(D) Cleavage of mouse GSDMD (mGSDMD) by mouse neutrophil lysate. FLAGmGSDMD was overexpressed in HEK293T cells and was incubated with mouse neutrophil lysate for the times indicated. 
(E) hGSDMD cleavage by human neutrophil lysate was mediated by serine proteases. The cleavage of FLAG-hGSDMD by caspase-1 or human neutrophil lysate was conducted as described in Figure 4C in the presence of indicated inhibitors.

(F) The cleavage of mGSDMD by mouse neutrophil lysate in the presence of caspase or serine protease inhibitor.

(G) The cleavage of hGSDMD by serine proteases. FLAG-hGSDMD was overexpressed in HEK293T cells. Cell lysates containing recombinant FLAG-hGSDMD were incubated with human recombinant caspase 1 (100 units), PR3 ( $2 \mu \mathrm{g})$, ELANE ( $2 \mu \mathrm{g})$, or cathepsin G (2 $\mu \mathrm{g})$ at $37^{\circ} \mathrm{C}$ for $30 \mathrm{~min}$ in a 6-well plate. The positions of the FL- and ELANE-cleaved hGSDMD are indicated.

$(\mathrm{H})$ The cleavage of hGSDMD by human neutrophil lysate in the presence of the indicated number of ELANE-specific inhibitors.

(I) The cleavage of mGSDMD by mouse ELANE. FLAG-mGSDMD was overexpressed in HEK293T cells and incubated with the indicated proteases. ELANE enzymatic activity was determined using an ELANE-specific substrate following the manufacturer's protocol.

(J) The cleavage of mGSDMD by ELANE-deficient neutrophil lysate. Disruption of ELANE was confirmed by western blotting with ELANE antibody.

(K) The cleavage of mGSDMD by caspase-1/11-deficient neutrophil lysate.

(L) Total peritoneal cell and peritoneal neutrophil numbers in E. coli-challenged WT and caspase-1/11-deficient mice. Total neutrophil content in the lavage fluid was calculated by FACS analysis.

Data shown at each time point are means \pm SDs of four mice. NS, not significant; $p>0.05$ versus WT. 
A

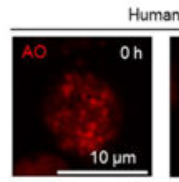

uman
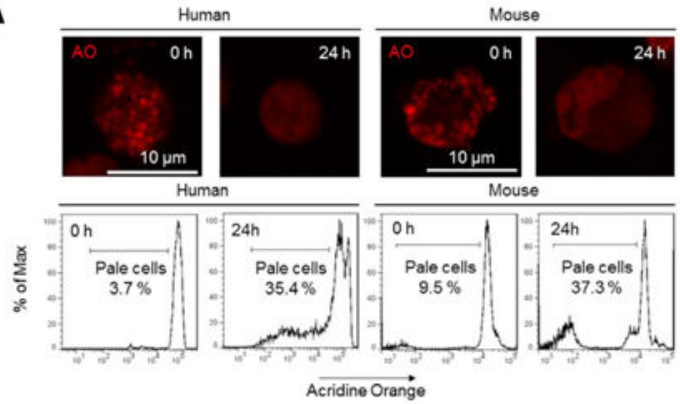

C

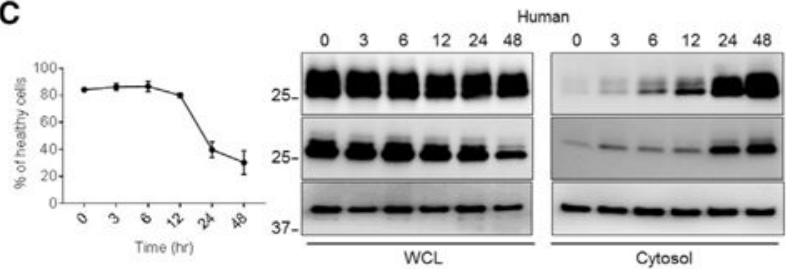

E

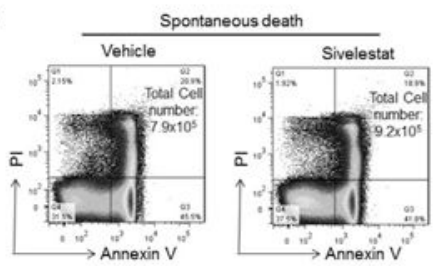

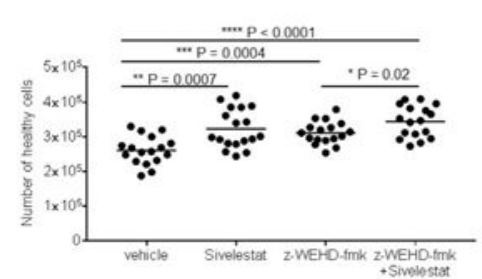

B
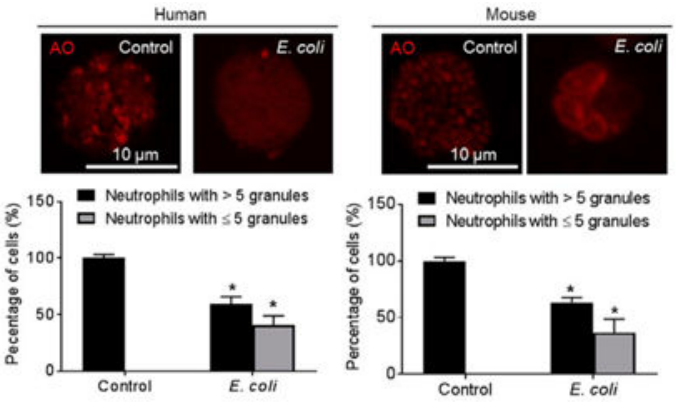

D Human
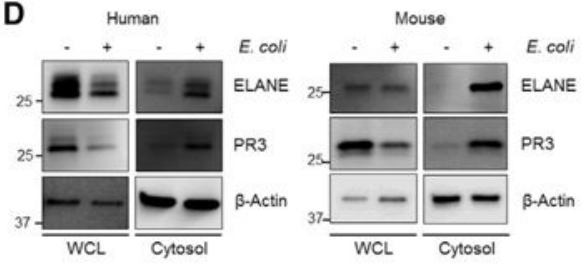

$\mathbf{F}$

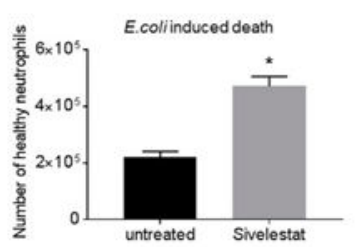

G

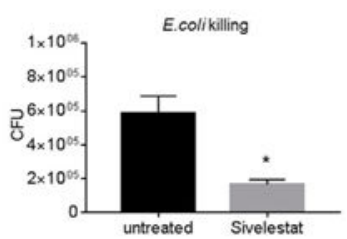

Figure 5. Lysosomal Membrane Permeabilization Induces ELANE Release into the Cytosol and the Subsequent GSDMD Cleavage

(A) Neutrophil lysosomal membrane permeabilization (LMP) was assessed using the acridine orange (AO) uptake assay after $24 \mathrm{hr}$ of culture. Shown are representative images and FACS plots of three independent experiments.

(B) E. coli-induced LMP. Mouse or human neutrophils were cultured with opsonized E. coli (1:5 ratio) for $30 \mathrm{~min}$. Cells containing $\geq 5$ granules and cells containing $<5$ granules are calculated. Shown are representative images of three independent experiments.

(C) PR3 and ELANE protein expression in whole-cell lysates (WCL) and the cytosolic fraction of fresh and aging neutrophils. Shown are representative blots of three independent experiments.

(D) PR3 and ELANE protein expression in neutrophils treated with E. coli. Human and mouse neutrophils were cultured with opsonized E. coli (1:5 ratio) for $60 \mathrm{~min}$. Shown are representative blots of three independent experiments.

(E) Spontaneous neutrophil death in the presence of ELANE, caspase-1/4/5-specific inhibitors, or all of these. Human primary neutrophils were isolated and cultured in the presence of ELANE-specific inhibitor sivelestat $(1 \mu \mathrm{g} / \mathrm{mL})$, caspase-1/4/5-specific inhibitor $\mathrm{z}$-WEHD-fmk $(10 \mu \mathrm{M})$, or all of these for $26 \mathrm{hr}$. All of the values represent means \pm SDs. (F) E. coli-induced neutrophil death in the presence of ELANE inhibitor. Human neutrophils were cultured with opsonized $E$. coli (1:5 ratio) in the presence of sivelestat $(1 \mu \mathrm{g} / \mathrm{mL})$ for $60 \mathrm{~min}$. All of the values represent means \pm SDs of three experiments.

(G) In vitro killing of $E$. coli by aged human neutrophils. Human primary neutrophils were isolated and cultured in the presence of ELANE-specific inhibitor sivelestat $(1 \mu \mathrm{g} / \mathrm{mL})$ for 
$26 \mathrm{hr}$. The cultured aged neutrophils were then incubated with $E$. coli for $1 \mathrm{hr}$. In vitro bacterial killing capabilities were reflected by the decrease in CFU after indicated incubation periods.

All of the values represent means \pm SDs of three experiments. *p $<0.05$ versus control. 
A
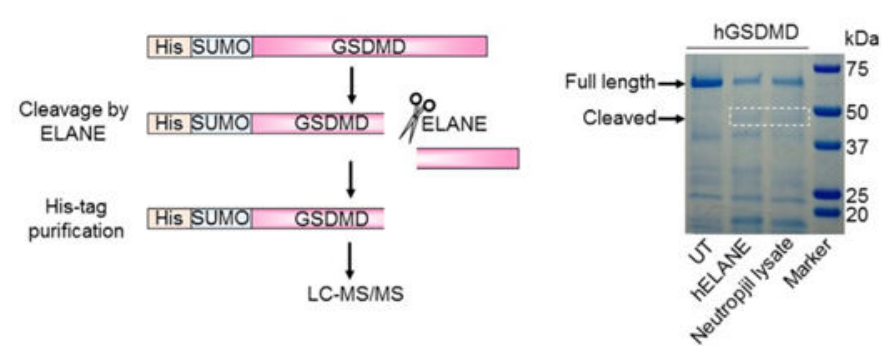

D

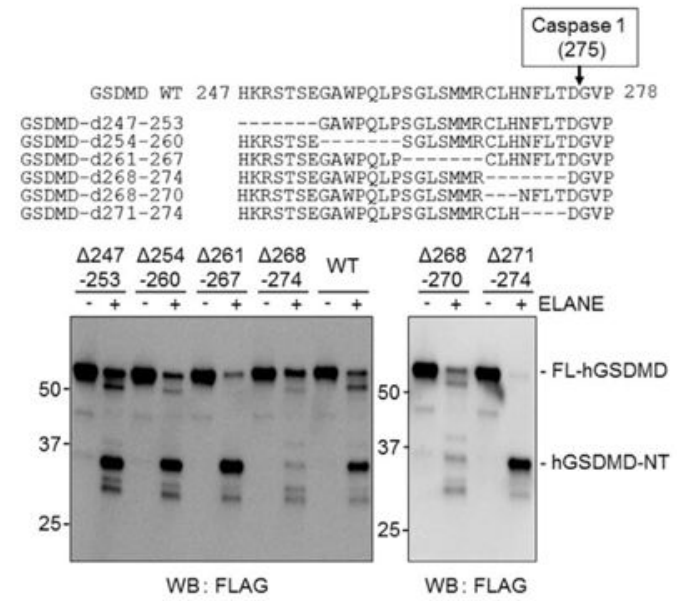

$\mathbf{F}$
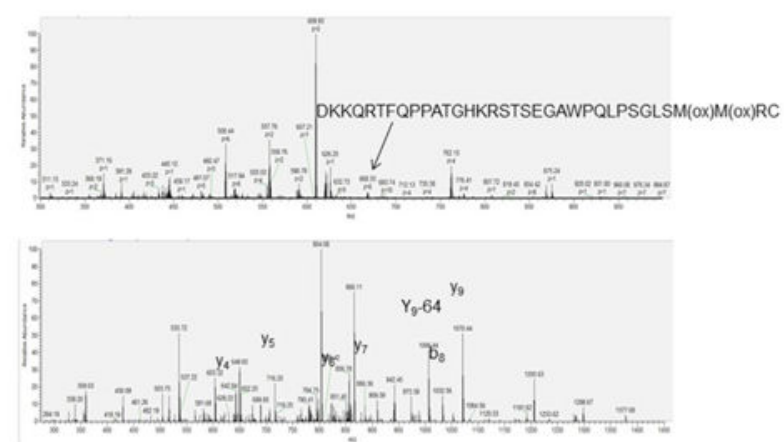

C

Trypsin Digestion: _ peptides derived from ELANE-cleaved GSDMD

- peptides derived from full length GSDMD MGSAYRRVVR RWVOELDHGG EYIFVTSLOS STOFOPYCLV VRKPSSSMFW 50 KPRYKCVILS IKDILEPDAA ERDVORGRSF HFYDAMDGOI OGSVELAAPG 100 QAKIAGGANV SDSSSTBMIV YSLSVDPNTW QTLLRERHLR OPEHKVL OOL 150 RSRGDEVYVV TEVLOTOKEV EVTRTHKREG SGRY SLPOAT CLQGEOOGHL 200 SOKKIVTIPS GSTIAPRVAO LVIDSDLDVL, LFPDKKORTF OPPATCHKRS 250

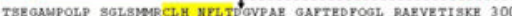
LELLDRELCO LLLECLEGVL ; PDOLALPALE gaLEOGCQLG PVERLDGPAG 350

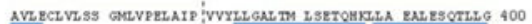
PLELVGSLLE QSAPNOERBT MSLPPOLLON SWEZGAPANV LLDECGLRLO 450 EDTPHVCWEP OROGMCALI Y iASLALLGGLS QEPH

$$
\text { Putative ELANE sites }
$$

E
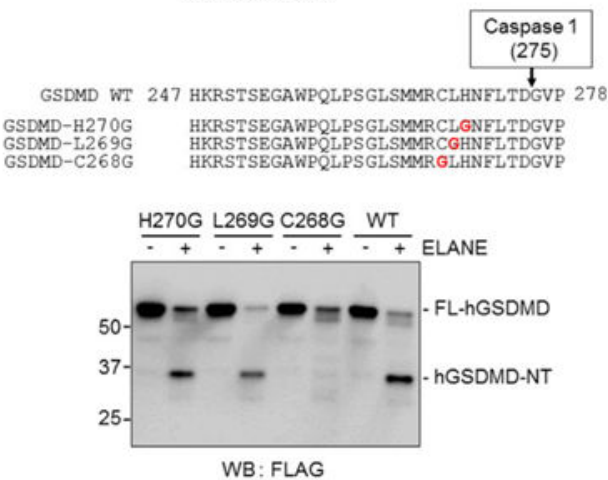

G

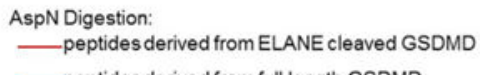

_-peptides derived from ELANE cleaved GSD

MOSAPERVVR RVVQRLPHGG EFIPVTSLOS STGPOP YCLV VRKP SSSWRW 50

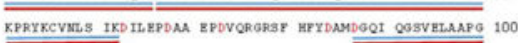
OAKIAGGAV SDSSSTSMNV YSLSVDPHTW OTLLIIERILR OPEHKVLCOL 150 RSRGDHVIVV TEVLOTOKEV EVTRTHKREG SGRPSLPGAT CLOGEGQGHL 200 SOKKTVTIPS GSTLAFRVAO LVIDSDLDVL LFPDKKQRTP OPPATOHKRS 250

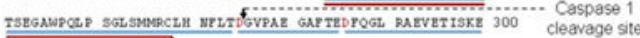

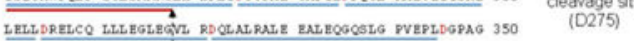

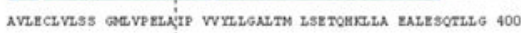

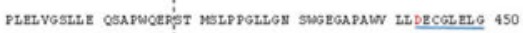
EDTPHVCUEP EROGRMCALY ASLALLSGLS QRPH

El.ANE deavage ste (C268)

H

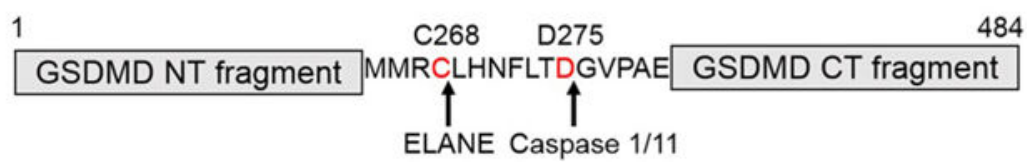

Figure 6. ELANE Cleaves GSDMD Upstream of the Caspase Cleavage Site

(A) Schematic ofGSDMD cleavage by ELANE and the strategy for identifying the cleavage site by mass spectrometry (MS).

(B) Recombinant human His-SUMO-GSDMD was incubated with ELANE or human neutrophil lysates at $37^{\circ} \mathrm{C}$ for $30 \mathrm{~min}$ and subjected to SDS-PAGE followed by colloidal blue staining. The FL and ELANE-cleaved GSDMD-NT fragment were trypsin digested and analyzed by MS. 
(C) Trypsin-digested peptides derived from the FL (blue lines) and ELANE-cleaved (red lines) GSDMD are underlined. The putative ELANE cleavage sites are highlighted. (D) The cleavage of hGSDMD deletion mutants by ELANE. The FLAG-tagged hGSDMD deletion mutants were overexpressed in HEK293T cells. ELANE-mediated hGSDMD cleavage was carried out and analyzed as described in Figure 4C.

(E) The cleavage of hGSDMD point mutants by ELANE. Results are representative of four independent experiments.

(F) MS analysis of the ELANE cleavage site in hGSDMD was conducted using AspNdigested samples. Top, mass spectrum of oxidized AspN-peptide generated through the cleavage. Bottom, MS/MS spectrum of the corresponding peptide showing the fragment ions detected and used for protein identification.

(G) MS analysis identifies C268 as the ELANE cleavage site in hGSDMD. AspN-digested peptides derived from the FL (blue lines) and ELANE-cleaved (red lines) GSDMD are underlined.

(H) ELANE- and caspase-1-cleaved hGSDMD at different sites. 


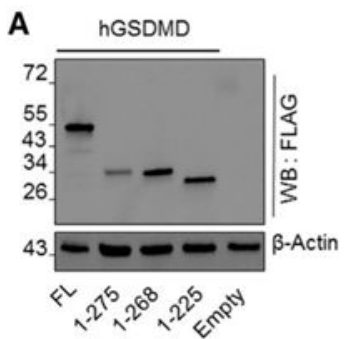

B

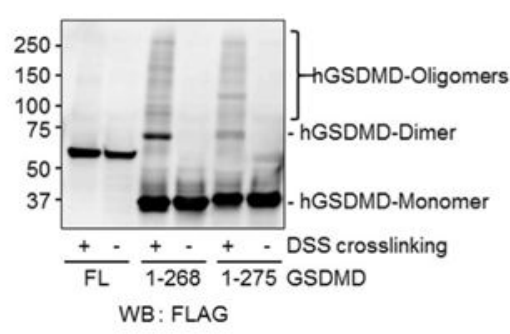

C

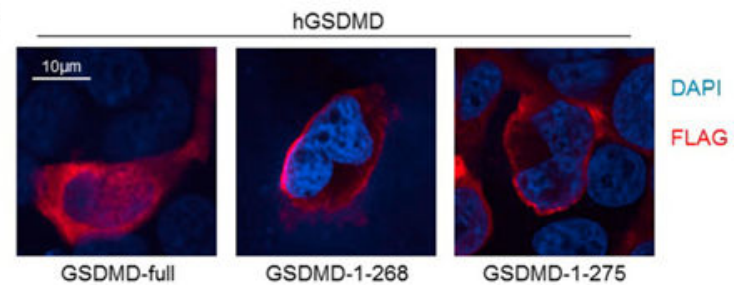

E
GSDMD-1-268

GSDMD-1-275
D

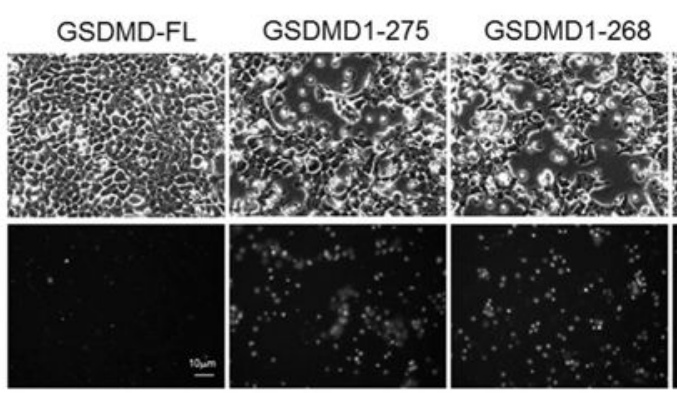

GSDMD1-225

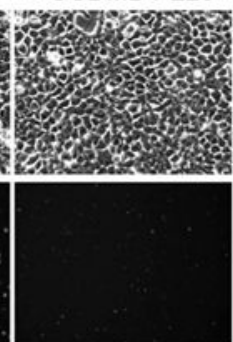

Empty

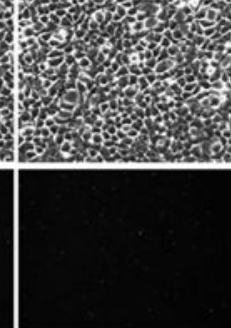

Figure 7. ELANE Cleaves GSDMD to Generate a Smaller but still Biologically Active GSDMDeNT Fragment

(A) Expression of FLAG-tagged FL, ELANE-cleaved (hGSDMD-eNT or 1-268), and caspase-cleaved (hGSDMD-cNT or 1-275) human GSDMD in HEK293T cells. The cells were lysed $24 \mathrm{hr}$ post-transfection.

(B) GSDMD protein oligomerization. The cells expressing the indicated hGSDMD protein were lysed with lysis buffer with or without disuccinimidyl suberate crosslinking reagent (1 $\mathrm{mg} / \mathrm{mL}$ ) and incubated at room temperature for $30 \mathrm{~min}$. The reaction was quenched by 0.1 M Tris, $\mathrm{pH} 7.4$.

(C) Subcellular localization of recombinant hGSDMD in 293T cells. Shown are representative images of three independent experiments.

(D) Cell morphology of transfected cells was observed by bright field microscopy $24 \mathrm{hr}$ post-transfection (see also Movies S1, S2, S3, and S4). PI staining was conducted to assess cell death. Shown are representative images of three independent experiments.

(E) Cytotoxicity was measured using a lactate dehydrogenase (LDH) cytotoxicity assay. All of the values represent means \pm SDs of three independent experiments. 